\title{
Correspondence
}

\section{Treatment of common recalcitrant warts with topical formic acid \\ Dear Editor,}

In 2001, Bhat et al. ${ }^{1}$ published in this journal the results of an open, placebo-controlled, nonrandomized trial on the treatment of common warts with topical $85 \%$ formic acid.

In the summer of 2016, a "pen" (Endwarts pen ${ }^{\circledR}$ ) containing formic acid was marketed in Italy. We present the results of an open, sponsor-free study on the efficacy and tolerability of this pen in patients with common recalcitrant warts. Diagnosis of warts was clinical. The case list is made up of 23 Caucasian, immunocompetent patients (17 males and 6 females, aged 18-33 years [mean age: 23.2 years]), with a total of 93 warts located on the face, elbows, hands, and knees. One patient was pregnant. All patients were previously, although unsuccessfully, treated at other centers with at least two different therapeutic modalities: curettage (18 patients), salicylic acid (16 patients), cryotherapy (13 patients), electrodesiccation (11 patients), laser therapy (8 patients), imiquimod (5 patients), and surgical excision (1 patient). Although the guidelines of the manufacturer suggest an application time of 1 second, the pen was applied on each wart for 30 seconds, twice weekly for 6 weeks. The choice of this duration was owing to the fact that in a previous group of eight patients, treated with an application time of 1 second, only mild improvement of the lesions was observed. No other topical or systemic drugs were used. No physical or surgical treatments were used.

Eighteen of 23 patients were considered as evaluable: five patients were lost to follow-up (these patients were affected by a total of 9 warts: $93-9=84$ warts). At the end of the treatment, a total number of 36 warts disappeared (36 out of $84=42.8 \%$ ). Complete remission was observed in eight patients who had a range of 1-4 warts before the beginning of the treatment. No differences with regard to therapeutic response between warts in different locations were observed. One patient reported a mild burning sensation on the area of application, but it was unnecessary to stop the treatment. No cases of pain were reported.

Formic acid is a carboxylic acid. It appears as a colorless liquid with a typical smell. In nature, it is found in red ants (Formica rufa) and stinging nettles (Urtica dioica).

To our knowledge, three clinical studies on the treatment of common warts with formic acid have been published. ${ }^{1-3}$ In the first one, 50 patients were treated with $85 \%$ formic acid and 50 patients received placebo (water), using a needle puncture technique every other day. Ninety-two percent of patients who were treated with formic acid showed complete disappearance of warts after 3-4 weeks of treatment, compared to $6 \%$ in the placebo group. ${ }^{1}$ A case control, double-blind trial was performed in 79 students, aged 12-17 years, with common warts located on the scalp, hands, trunk, and feet. One group of 42 patients was treated with $85 \%$ formic acid; the control group consisted of 37 patients who were treated with water as placebo. Applications were done on alternate days for 4 weeks. The total number of applications was limited to 12, after which the treatment was considered a failure. In case and control groups, the average number of applications required for the warts to disappear was $5.9 \pm 3.8$ vs. $11.1 \pm 2.6$, respectively. The efficacy of the treatment, assessed at the end of the first month as complete or partial remissions, was $81 \%$ vs. $9.5 \%$ and $11 \%$ vs. $2.7 \%$, respectively. ${ }^{2}$ An additional placebo-controlled trial was performed in 2010 . A total of 34 patients received $85 \%$ formic acid on their lesion on one side of the body and distilled water as placebo on the other side of the body, every other day, using a needle puncture technique. Follow-up was every 2 weeks up to 3 months. Ninety-one percent of patients who were treated with formic acid showed complete disappearance of warts after the follow-up period, compared to $10 \%$ in the placebo group. ${ }^{3}$

Mechanisms of action of formic acid in warts remain unknown. According to some authors, ${ }^{2}$ it acts like formalin by dehydrating and finally destroying the infected tissue. This would be demonstrated by the fact that the warts become slightly white in color, followed by desquamation of the superficial layers. In all studies, it was stated that the treatment with formic acid is inexpensive, easy, fast, safe (it can also be used in pregnancy, as in our patient), and painless. Adherence to treatment and compliance are therefore very high. ${ }^{1-3}$ However, one case of a deep full thickness burn from formic acid application was reported. This patient developed a burn over the proximal interphalangeal joint of her fifth finger, after inappropriate application of formic acid. The burn required debridement and reconstruction using a subcutaneous flap from the adjacent finger (a reverse cross finger flap). ${ }^{4}$

The results of our study may be summarized as follows: (i) formic acid was able to cure more than $42 \%$ of common warts that were previously resistant to a number of pharmacologic and surgical treatments. This percentage can be considered, in our personal clinical experience, as good: the very high percentage of complete remissions reported by two of the previously cited trials (92\% and $91 \%$, respectively) is very surprising; ${ }^{1,3}$ (ii) no important side effects were reported or observed, and as previously mentioned, only one patient reported mild irritation.

A controlled clinical study is necessary to confirm or disprove our results. 


$$
\begin{array}{r}
\text { Rossana Schianchi }{ }^{1}, \mathrm{MD} \\
\text { Michela Brena }^{2}, \mathrm{MD} \\
\text { Stefano Veraldi }{ }^{2, *}, \mathrm{MD}
\end{array}
$$

${ }^{1}$ Dermatological Center in Milan (C.D.M.), Milan, Italy ${ }^{2}$ Department of Pathophysiology and Transplantation, Università degli Studi di Milano, I.R.C.C.S. Foundation, Cà

Granda Ospedale Maggiore Policlinico, Milan, Italy

*E-mail: stefano.veraldi @unimi.it

Funding: None.

Conflicts of interest: The authors have no conflict of interest to declare.

doi: $10.1111 / \mathrm{ijd} .13811$

\section{References}

1 Bhat RM, Vidya K, Kamath G. Topical formic acid puncture technique for the treatment of common warts. Int $\mathrm{J}$ Dermatol 2001; 40: 415-419.

2 Shamsadini S, Baghery MH. Treatment of warts with topical formic acid. Iran J Med Sci 2005; 30: 199.

3 Faghighi G, Vali A, Radan M, et al. A double-blind, randomized trial of local formic acid puncture technique in the treatment of common warts. Skinmed 2010; 8: 70-71.

4 Tong E, Dorairaj J, O'Sullivan JB, et al. Deep full thickness burn to a finger from a topical wart treatment. Ir Med J 2015; 108: 283-284. 